EPJ Web of Conferences 19, 01005 (2012)

DOI: $10.1051 /$ epjconf/20121901005

(C) Owned by the authors, published by EDP Sciences, 2012

\title{
Simulating the Milky Way is hard
}

\author{
G. Stinson ${ }^{1,3, a}$ and C. Brook ${ }^{2,3}$ \\ ${ }^{1}$ Max-Planck-Institut für Astronomie, Königstuhl 17, 69117 Heidelberg, Germany \\ ${ }^{2}$ Grupo de Astrofísica, Departamento de Fisica Teorica, Modulo C-15, Universidad Autónoma \\ de Madrid, 28049 Cantoblanco, Spain \\ 3 Jeremiah Horrocks Institute, University of Central Lancashire, Preston PR1 2HE, UK
}

\begin{abstract}
The formation of the Milky Way in the currently accepted concordance cosmology presents a couple of pitfalls. Mostly, these involve the much reported overcooling problem. If gas is allowed to cool, it will condense into massive central concentrations unlike any observed in real galaxies. These concentrations are characterized by a high central peak in the rotation curve and a rise in the surface brightness profile much steeper than the exponentially increasing disk. Such galaxies may contain no disk component and form many more stars than predicted by abundance matching studies.
\end{abstract}

\section{INTRODUCTION}

There are four major problems with the galaxies formed in fully cosmological simulations:

- They form too many stars [1]

- Their rotation curves are too centrally peaked

- They have high central surface brightnesses

- Bulge-to-total ratios are high for every $\mathrm{L}^{*}$ galaxy ever simulated

The evidence for simulations forming too many stars comes from recent studies using the abundance matching technique. To lay the groundwork for this technique, Conroy et al (2006) rank ordered halos by total halo mass from collisionless simulations and then did a rank ordering of galaxies based on their stellar mass from the Sloan Digital Sky Survey [2]. The halos and galaxies were then divided in several mass bins. Conroy et al (2006) found that the correlation functions between halos in certain mass bins were well matched by the correlation functions of galaxies in corresponding stellar mass bins [2]. Several groups used this abundance matching technique to compare total halo masses with galaxy stellar masses $[1,3,4]$. Similar comparisons of stellar mass and total halo mass have been done based on satellite dynamics [5, 6] and weak lensing [7]. For a comparsion of all these techniques, please see Figure 11 of Behroozi et al (2010) [4]. Each method shows a good level of correspondence. Guo et al (2010) and Sawala et al (2011)showed that nearly all the simulations of galaxy formation have formed many more stars than abundance matching predicts $[1,8]$.

The excess star formation and the other three problems are all symptoms of the overcooling catastrophe. Fully cosmological numerical galaxy simulations consistently contain an excess of low angular momentum material [9-11]. This is evident in the central peak in rotation curves in simulated galaxies as well as in their surface brightness profiles. van den Bosch et al (2002) showed that this excess low angular momentum material is the natural result of gas being accreted with the same angular momentum profile as dark matter [12]. Consequently, this low angular momentum material

ae-mail: stinson@mpia.de

This is an Open Access article distributed under the terms of the Creative Commons Attribution-Noncommercial License 3.0, which permits unrestricted use, distribution, and reproduction in any noncommercial medium, provided the original work is properly cited. 


\section{EPJ Web of Conferences}

needs to be removed from the center of the system. Dutton et al (2009) showed that as simple model for supernova feedback can remove the central concentration analytically [13]. Brook et al (2011) showed that high resolution simulations of dwarf galaxies can accomplish this task [14]. However, reproducing the resolution of those simulations in a Milky Way mass galaxy are currently infeasible with current computers.

There is general agreement that feedback is the way to solve these problems $[15,16]$. Two methods are commonly used. One is kinetic feedback that adds velocity kicks to gas particles to remove them from the inner regions of galaxy disks. The other is thermal feedback in which stars simply heat gas particles and allow the PdV work of the particles push other gas out of the way. Since stars form in dense regions, the cooling times are short. There are few other methods available to simulators to push gas out of the central regions of galaxies.

Kinetic feedback is another alternative. It was described in Navarro \& Steinmetz (1997) [17], and many simulations currently use the method described in Springel et al 2003 [16, 18-22]. That method ejects a gas particle or two every time another gas particle gets turned into a star. Particles are given velocities of either an observationally motivated fixed value like $400 \mathrm{~km} \mathrm{~s}^{-1}$ or a value corresponding to $v_{\text {esc }}$ of the halo to which that particle is a member. Springel et al (2005) found that the removal of particles and high cooling rates lead to dramatically unstable disks when the disks were dominated by gas, so they fixed the equation of state to $P \sim \rho^{2}$ in high density regions, which provides significantly more pressure support and thus disk stability than the $P \sim \rho^{5 / 3}$ ideal gas equation of state used commonly in simulations [23].

Resolution remains a problem for modeling feedback. State-of-the-art simulations model galaxies with a million gas particles inside $r_{v i r}$. This means that each gas particle has a mass of $10^{5} \mathrm{M}_{\odot}$ in the case of an $\mathrm{L}^{*}$ galaxy such as the Milky Way. Stars are formed with some fraction of this mass, so each star particle represents more than $10^{4} \mathrm{M}_{\odot}$. In reality, such a mass of stars would be comprised of multiple energy sources each isotropically irradiating a small part of the interstellar medium. We know that the fraction of gas converted to stars is small during the free fall time of the gas [24].

In addition to mass resolution, it is difficult to spatially resolve feedback processes. For a halo to dynamically relax over the age of the Universe, the gravitational softening length cannot be smaller than $r_{v i r} / \sqrt{N_{v i r}}$ [25]. For $10^{6}$ particles within $r_{v i r}$, this means softening lengths of $200 \mathrm{pc}$. Molecular clouds are $10 \mathrm{pc}$ and all the shocks and intricate features inside them are much smaller.

One of the reasons that energetic feedback is necessary in simulations is the limited time resolution. The limited time resolution contributes to the cooling catastrophe. Since fully cosmological simulations must run for $13.7 \mathrm{Gyr}$, the age of the Universe, maximizing the length of timesteps to limit the number of timesteps that must be calculated is desirable. Timesteps must be small enough to resolve the gravitational and hydrodynamics. The dynamical condition for timesteps is $\Delta t=\sqrt{\epsilon / a}$, where $\epsilon$ is the gravitational softening length and $a$ is a particle's acceleration. The most accelerated particles are typically at the centers of galaxies where their orbits enclose $10^{10} \mathrm{M}_{\odot}$ inside $2 \mathrm{kpc} . a=G M / R^{2}$, so $\Delta t \sim 10^{4}$ yrs. The hydrodynamic Courant condition is $\Delta t=h_{s m} / c_{s}$. Hot, dense gas has the shortest timesteps.

One of the major issues with SPH is that the primary smoothed quantity is density, which is averaged over a selected number of nearest neighbors $\left(N_{\text {smooth }}\right)$. Typically, $N_{\text {smooth }}>32$. In the simulation with $10^{6}$ particles inside $r_{v i r}$, that is $3.2 \times 10^{6} \mathrm{M}_{\odot}$ inside $500 \mathrm{pc}$, which is more gas than would ideally be heated. If all the gas is not heated, then the cooler particles at high density will dominate the density calculation. The cooling times for such high density particles from $10^{6} \mathrm{~K}$ are shorter than the typical dynamical times for the particles, so the hot particles never have a chance to adiabatically expand.

A couple of solutions have been proposed to deal with this issue. Ritchie and Thomas (2001) smooth over pressure instead of density [26]. In the case of one $10^{6} \mathrm{~K}$ particle nearby $3110^{4} \mathrm{~K}$ particles at $n=10 \mathrm{~cm}^{-3}$ the resulting density is only a factor of 3 lower than using normal density smoothing, so the cooling time is only lengthened from $10^{4} \mathrm{yr}$ to $3 \times 10^{4} \mathrm{yr}$, which is still shorter than the typical dynamical time. Scannapieco et al (2006) described a scheme to calculate densities of high and low 

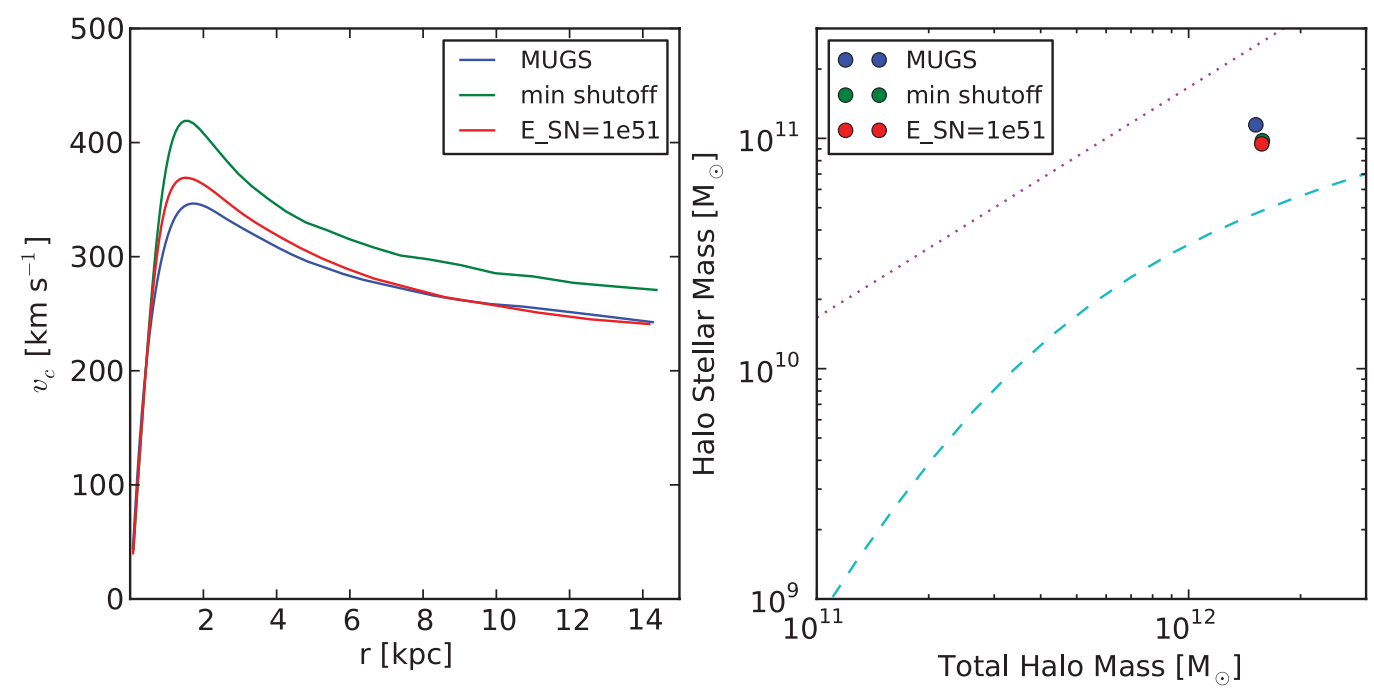

Figure 1. Two common symptoms of overcooling in galaxy formation simulation of a Milky Way mass galaxy: a centrally peaked rotation curve and a higher stellar mass than what is predicted by abundance matching studies. These plots show that increasing feedback is not guaranteed to fix these problems in Milky Way mass galaxies.

entropy particles separately [27]. This method has shown some promise at forming disks [28, 29] and dwarf galaxies [8,30], but It is difficult to know when to move such particles from the low to the high entropy classification and back.

Most cosmological SPH simulations only inject energy from supernovae explosions [11, 28, 31]. Such simulations ignore the early radiation generated by massive stars. Hopkins et al (2011) introduced a scheme that uses this energy [32]. The scheme uses extremely large numbers of particles.

\section{STAR FORMATION AND FEEDBACK}

The simulations shown here use a common star formation recipe described in Stinson et al (2006) [33]. Stars feed both energy and metals back into the interstellar medium gas surrounding the region where they formed. Supernova feedback is implemented using the blastwave formalism described in Stinson et al (2006) [33]. In this, Type II supernovae are assumed to explode due to the core collapse at the end of lifetime of stars greater than $8 \mathrm{M}_{\odot}$. In MUGS, supernovae deposited $4 \times 10^{50} \mathrm{erg}$ of energy into the surrounding medium. Here, we try increasing the feedback, so that $E_{S N}$ is $1 \times 10^{51} \mathrm{erg}$. It is assumed that the rapid cooling will take care of reducing the efficiency of this feedback. The MUGS simulations utilize a stochastic feedback scheme that requires each episode of energy input to include at least 20 supernovae worth of energy. Here, we reverting back to the Stinson et al (2006) method that released limited amounts of energetic feedback continuously.

\section{RESULTS}

Figure 1 shows two common symptoms of overcooling in galaxy formation simulation of a Milky Way mass galaxy: a centrally peaked rotation curve and a higher stellar mass than what is predicted by abundance matching studies. Two new simulations of the same initial conditions modeled with stronger supernova feedback are shown in comparison with the MUGS simulations. The models with stronger feedback show a moderate decrease in the total amount of star formation, but not enough to lower the star formation to the amount seen in abundance matching. The higher feedback simulations also create 
more mass in the centers of galaxies than MUGS did, causing an even higher central peak in the rotation curve. These plots show that increasing feedback is not guaranteed to fix the problems we find when simulating Milky Way mass galaxies.

\section{CONCLUSIONS}

Simulations have trouble reproducing Milky Way mass disk galaxies because of overcooling. Much angular momentum is lost and gas cools into the centers of simulated galaxies where it forms a lot of stars. Such a galaxy exhibits a rotation curve that steeply rises to the center and more star formation than is predicted by a range of observations of halo and stellar masses. We attempted to increase the supernova feedback in some simulations and did not find a resolution to these problems, so we continue searching for a physical mechanism that will correctly form a Milky Way mass galaxy.

\section{References}

[1] Q. Guo, S. White, C. Li, M. Boylan-Kolchin, MNRAS 404, 1111 (2010), 0909.4305

[2] C. Conroy, R.H. Wechsler, A.V. Kravtsov, ApJ 647, 201 (2006), arXiv : astro-ph/0512234

[3] B.P. Moster, R.S. Somerville, C. Maulbetsch, F.C. van den Bosch, A.V. Macciò, T. Naab, L. Oser, ApJ 710, 903 (2010), 0903.4682

[4] P.S. Behroozi, C. Conroy, R.H. Wechsler, ApJ 717, 379 (2010), 1001.0015

[5] S. More, F.C. van den Bosch, M. Cacciato, H.J. Mo, X. Yang, R. Li, MNRAS 392, 801 (2009), 0807.4532

[6] S. More, F.C. van den Bosch, M. Cacciato, R. Skibba, H.J. Mo, X. Yang, MNRAS 410, 210 (2011), 1003.3203

[7] R. Mandelbaum, C. Li, G. Kauffmann, S.D.M. White, MNRAS 393, 377 (2009), 0806.4089

[8] T. Sawala, Q. Guo, C. Scannapieco, A. Jenkins, S. White, MNRAS 413, 659 (2011), 1003.0671

[9] J.F. Navarro, W. Benz, ApJ 380, 320 (1991)

[10] F. Governato, L. Mayer, J. Wadsley, J.P. Gardner, B. Willman, E. Hayashi, T. Quinn, J. Stadel, G. Lake, ApJ 607, 688 (2004)

[11] G.S. Stinson, J. Bailin, H. Couchman, J. Wadsley, S. Shen, S. Nickerson, C. Brook, T. Quinn, MNRAS 408, 812 (2010)

[12] F.C. van den Bosch, T. Abel, R.A.C. Croft, L. Hernquist, S.D.M. White, ApJ 576, 21 (2002), arXiv:astro-ph/0201095

[13] A.A. Dutton, F.C. van den Bosch, MNRAS 396, 141 (2009), 0810.4963

[14] C.B. Brook, F. Governato, R. Roškar, G. Stinson, A.M. Brooks, J. Wadsley, T. Quinn, B.K. Gibson, O. Snaith, K. Pilkington et al., MNRAS pp. 595-+ (2011), 1010.1004

[15] C. Scannapieco, P.B. Tissera, S.D.M. White, V. Springel, MNRAS 389, 1137 (2008), 0804.3795

[16] J. Schaye, C. Dalla Vecchia, C.M. Booth, R.P.C. Wiersma, T. Theuns, M.R. Haas, S. Bertone, A.R. Duffy, I.G. McCarthy, F. van de Voort, MNRAS 402, 1536 (2010), 0909.5196

[17] J.F. Navarro, M. Steinmetz, ApJ 478, 13 (1997), arXiv : astro-ph/9605043

[18] V. Springel, L. Hernquist, MNRAS 339, 289 (2003), arXiv: astro-ph/0206393

[19] K. Nagamine, V. Springel, L. Hernquist, MNRAS 348, 435 (2004), arXiv : astro-ph/0305409

[20] B.D. Oppenheimer, R. Davé, MNRAS 373, 1265 (2006), arXiv : astro-ph/0605651

[21] T. Okamoto, C.S. Frenk, MNRAS 399, L174 (2009), 0909.0262

[22] R. Davé, B.D. Oppenheimer, K. Finlator, MNRAS 415, 11 (2011), 1103. 3528

[23] V. Springel, L. Hernquist, ApJ 622, L9 (2005)

[24] M.R. Krumholz, J.C. Tan, ApJ 654, 304 (2007), arXiv : astro-ph/0606277

[25] C. Power, J.F. Navarro, A. Jenkins, C.S. Frenk, S.D.M. White, V. Springel, J. Stadel, T. Quinn, MNRAS 338, 14 (2003), arXiv: astro-ph/0201544 
[26] B.W. Ritchie, P.A. Thomas, MNRAS 323, 743 (2001), arXiv: astro-ph/0005357

[27] C. Scannapieco, P.B. Tissera, S.D.M. White, V. Springel, MNRAS 371, 1125 (2006), arXiv: astro-ph/0604524

[28] C. Scannapieco, D.A. Gadotti, P. Jonsson, S.D.M. White, MNRAS 407, L41 (2010), 1001.4890

[29] C. Scannapieco, S.D.M. White, V. Springel, P.B. Tissera, MNRAS 417, 154 (2011), 1105.0680

[30] T. Sawala, C. Scannapieco, U. Maio, S. White, MNRAS 402, 1599 (2010), 0902.1754

[31] F. Governato, B. Willman, L. Mayer, A. Brooks, G. Stinson, O. Valenzuela, J. Wadsley, T. Quinn, MNRAS 374, 1479 (2007), arXiv: astro-ph/0602351

[32] P.F. Hopkins, E. Quataert, N. Murray, ArXiv e-prints (2011), 1101.4940

[33] G. Stinson, A. Seth, N. Katz, J. Wadsley, F. Governato, T. Quinn, MNRAS 373, 1074 (2006), astro-ph/0602350 